\title{
Recent Human-Specific Spreading of a Subtelomeric Domain
}

\author{
Sylvaine Monfouilloux,* Hervé Avet-Loiseau,† Valérie Amarger,* Ivan Balazs,‡ \\ Christine Pourcel, $\S$ and Gilles Vergnaud*, $, 1,1$
}

\begin{abstract}
* Laboratoire de Recherche en Génétique des Espèces and †U.F. Cytogénétique Hématologique, Institut de Biologie des Hôpitaux de Nantes, 9, Quai Moncousu, 44035 Nantes Cedex, France; łLifecodes Corporation, 550 W est Avenue, Stamford, Connecticut 06902; §Unité d'Immuno-Hématologie, Institut Pasteur, 25 rue du Dr Roux, 75724 Paris Cedex, France; and "Laboratoire de Génétique Moléculaire, Centre d'Etudes du Bouchet, B.P. 3, 91710 Vert le Petit, France
\end{abstract}

Received January 23, 1998; accepted May 1, 1998

The recent spreading of a subtelomeric region at nine different human chromosome ends was characterized by a combination of segregation analyses, physical mapping, junction cloning, and FISH investigations. The events occurred very recently in human genome evolution as demonstrated by sequence analysis of different alleles and the single location of the ancestral site at chromosome 17qter in chimpanzee and orangutan. The domain successfully colonized most $1 p, 5 q$, and $6 q$ chromosome ends and is also present at a significant frequency of $6 p, 7 p, 8 p, 11 p$, $15 q$, and $19 p$ ends. On 6qter, the transposed domain is immediately distal to the highly conserved, singlecopy gene PDCD2. ๑1998 Academic Press

\section{INTRODUCTION}

Humans and great apes are very highly similar at the DNA level despite the dramatic differences observed between these species. However, karyotype differences are visible, most of them associated with the ends of the chromosomes (Royle et al., 1994). A number of studies point to chromosome ends as hotspots for genome evolution, demonstrating differences even within the human population (Macina et al., 1995; Trask et al., 1998). Consequently the possibility that subtelomeric chromosomal rearrangements might have altered the regulation of one or more of these genes, which then had a significant impact on the evolution of our species, deserves investigation. For this purpose, and to develop efficient tools to study our recent evolution, human subtel omeric domains should be characterized in detail. I mmediately adjacent to the tel omere, in humans and in all eucaryotes investigated, begins a

Sequence data for this article have been deposited with the EMBL/ GenBank Data Libraries under Accession Nos. Y 13538 to Y 13550.

${ }^{1}$ To whom correspondence should be addressed at present address: Institut de Génétique et Microbiologie, Bat 400, Université Paris-Sud, 91405 Orsay Cedex, France. Telephone: +33 1691562 08. Fax: +33 1691566 78. E-mail: gilles@igmors.u-psud.fr. non-chromosome-specific subtelomeric domain. The size of the area located between the (TTAGGG) telomere repeats array and the chromosome-specific domain varies among chromosomes. It is only a few hundred basepairs long at human chromosome XpYpter (Baird and Royle, 1997), whereas it can be more than a hundred kilobases long at 16pter (Wilkie et al., 1991) or 4qter (van Deutekom et al., 1996). The transition from the subtelomeric, low-copy repeat domain to the chromosome-specific domain can be precisely defined, and in the few instances explored (16pter, 22qter, 4qter), genes are found at a high density starting a few kilobases proximal to the transition (van Deutekom et al., 1996; Flint et al., 1997b; Wong et al., 1997). However, the succession of subtelomeric layers usually makes in situ investigations very difficult to analyze and interpret. Here we report the detailed description of a very recent instance of a highly successful chromosome end spreading in human, which interestingly may be sufficiently simple to provide a new tool to investigate our recent evolution.

\section{MATERIALS AND METHODS}

Minisatellite probes. Minisatellite pAC265 was previously re ported (I p et al., 1989). Minisatellite CEB92 was obtained by screening a commercial cosmid library by hybridization with the synthetic tandem repeat probe (Vergnaud, 1989; Vergnaud et al., 1991). 16C27 is (AGCTACGGTGTGGACT) $n$.

Cell lines and DNA samples. Lymphoblastoid cell lines for individuals 1362-01, 1413-01, and 1413-02 and DNAs from the CEPH panel of 40 reference families were obtained from CEPH (Paris, France). Primate lymphoblastoid cell lines were purchased from ATCC (chimpanzee, Tank, Ref. 1847-CRL; orangutan, Puti, Ref. 1850-CRL).

Chromosome-specific cosmid libraries. High-density filters for human chromosome-specific cosmid libraries (chromosome 1, 6, and 17; library Nos. 112, 109, and 105) and individual clones were obtained from the Max-Planck Institute for Molecular Genetic (MPIMG). In addition, cosmid subclones generated from the halftelomeric Y ACs yRM 2158 (chromosome 6qter) and B22 (chromosome 5 qter) in the course of the development of chromosome-specific telomeric probes were a gift from J onathan Flint ( $\mathrm{NIH}$ and Institute of Molecular Medicine Collaboration, 1996). 


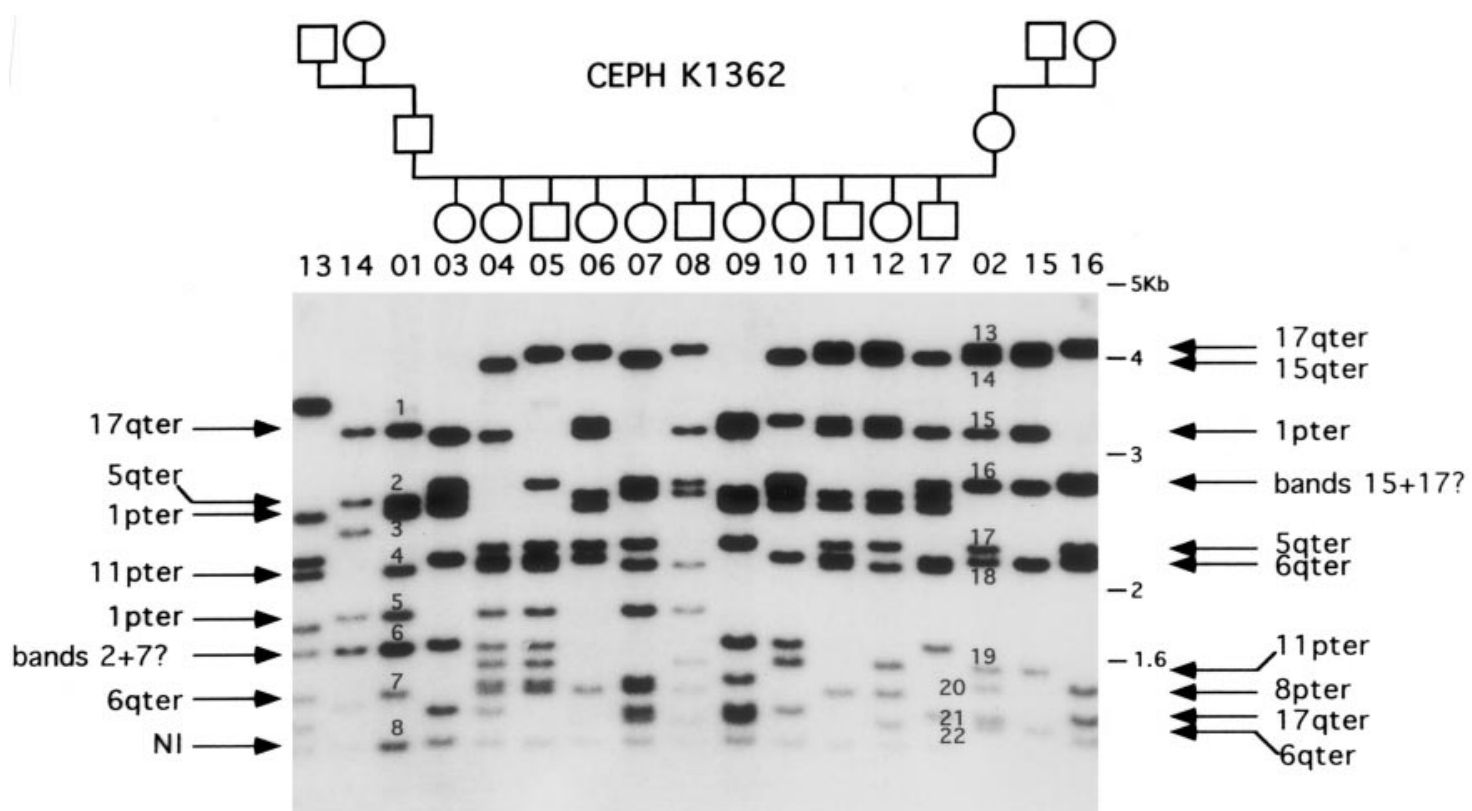

FIG. 1. Multilocus distribution of minisatellite locus CEB92. Southern blot analysis: Hybridization profile of CEB92 on CEPH family 1362 (Haell digest). The size of each band is indicated in kilobases. CE B92 reveals 8 paternal (I d136201) and 10 maternal (Id136202) bands. Segregation of 6 of the paternal alleles was easily read, and a significant linkage with one terminal CEPH database marker was found for each allele. The CEPH version 8.1 segregation database is accessible at http://www.cephb.fr. The localization deduced from two-point lod-score analysis is indicated at the left. For convenience, in all families analyzed, bands of paternal origin are numbered in the range 1 to 12, in decreasing size order, whereas maternal bands are numbered starting at 13. Paternal band 6 has a double intensity compared to other bands of similar size, suggesting a superposition of two bands. The segregation of the band intensity (no band, normal intensity, double intensity) in the progeny is compatible with band 6 being the superposition of two bands allelic of band 2 and band 7 . Band 8 has a double intensity in the father and is present at a normal intensity in all children, suggesting that it corresponds to one monomorphic locus (noninformative; NI). Nine segregating maternal alleles were linked to terminal markers with localization as indicated on the right. The maternal band 16, like paternal band 6, can be interpreted as the superposition of bands allelic to 15 and 17.

Hybridization procedures. Hybridization was done as described in Vergnaud et al. (1991) using a hybridization oven.

Minisatellite isolation. Candidate minisatellite-containing fragments were identified by digesting cosmid DNA simultaneously with two of the frequent cutters Alul, Haelll, and Hinfl. After separation by agarose gel electrophoresis, fragments above $1 \mathrm{~kb}$ were extracted and hybridized to Southern blots of a few CEPH families. Fragments giving a polymorphic hybridization profile were subcloned into the PUC 18 vector for sequencing.

Segregation analyses in the CEPH families. The segregation of informative bands detected by minisatellite probes in the $\mathrm{CEPH}$ reference families was compared to the CEPH database version 8.1 (http://www.cephb.fr) by two-point lod-score analysis using CRI MAP version 2.4 (Green et al., 1990).

Cosmid walking and contig building. Successive directed walking was performed by hybridization using cosmid end probes on library filters. Cosmid end probes were generated by PCR elongation of primers flanking the cloning site of the vector (the T3 and T7 primers were used for YACs subcloned into the SuperCos1 vector; PL1 5' ATACGACTCACTATAGGGAG $3{ }^{\prime}$ and PL2 5' ACATACGATTTAGGTGACAC $3^{\prime}$ were for cosmids based on the Lawrist4 vector (MPIMG-ICRF clones)). The reaction was done in $20 \mu \mathrm{l}$ containing $1 \mu \mathrm{g}$ of cosmid DNA; one primer at $0.5 \mu \mathrm{M} ; 25 \mu \mathrm{M}$ dATP, dGTP, and dTTP; $0.75 \mu \mathrm{M}$ dCTP (dNTPs from Pharmacia); $20 \mu \mathrm{Ci}$ of [ ${ }^{32} \mathrm{P}$ ]dCTP (ICN); and $2.5 \mathrm{u}$ of Taq polymerase (Pharmacia) in $45 \mathrm{mM}$ Tris, $\mathrm{pH} 9,11 \mathrm{mM}$ ammonium sulfate, $1.5 \mathrm{mM} \mathrm{MgCl} 2,6.7 \mathrm{mM}$ ß-mercaptoethanol, $4.5 \mu \mathrm{M}$ EDTA overlaid by oil. The reaction was performed with $30 \mathrm{cycles}$ of $94^{\circ} \mathrm{C}$ for $20 \mathrm{~s}, 55^{\circ} \mathrm{C}$ for $30 \mathrm{~s}$, and $72^{\circ} \mathrm{C}$ for $20 \mathrm{~s}$ in a PREM III thermocycler. Alternatively, when the resulting cosmid end probe was not appropriate for hybridization, usually because of the presence of a repetitive element, nearby restriction fragments were labeled by random priming.
DNA sequencing. Doublestranded DNA sequencing was done using the delta Taq sequencing kit (USB-Amersham) and end-labeled primers using kinase (New England Biolabs) and [ $\left.\gamma^{3}{ }^{33} \mathrm{P}\right] \mathrm{ATP}$ (Isotopchim). Sequence A1-4 was produced in parallel from different alleles by primer walking.

DNA sequence homol ogy searches. Homology searches were performed using the BLAST server and DNA databases hosted by NCBI (http://www.ncbi.nlm.nih.gov).

Fluorescence in situ hybridization (FISH). Cosmids were labeled with biotin by nick-translation (Bionick kit; BRL) and hybridized to metaphase spread chromosomes of lymphocytes from the different cell lines. The probe was denatured and annealed to human Cot-1 DNA (final concentration of $2 \mu \mathrm{g} / 200 \mathrm{ng}$ probe). Hybridization was performed at $37^{\circ} \mathrm{C}$ overnight. The hybridization signal was detected using an avidin/anti-avidin antibody detection system by a fluorescence microscope.

\section{RESULTS}

Linkage Analysis of the Two Highly Polymorphic Multicopy Minisatelites CEB92 (DNF92) and pAC365 (DNF24) Demonstrates Coassociation at a Number of Chromosome Ends

Minisatellites CEB92 and pAC365 were independently isolated in the course of searches for minisatellites within the human genome (I p et al., 1989; Vergnaud et al., 1991), and both detect patterns with multiple bands on Southern blots. The eight largest $\mathrm{CEPH}$ families were used for segregation analyses to 
TABLE 1

Segregation Data Analyses in the Eight Large CEPH Families

\begin{tabular}{|c|c|c|c|c|c|c|c|c|c|c|c|c|c|}
\hline Family & Child. & No. of bands (a) & 1pter & $5 q$ ter & 6 pter & 6qter & 7 pter & 8pter & 9qter & 11pter & $15 q$ ter & 17qter & ?? (b) \\
\hline & & DNF92 (CE B92) & & & & & & & & & & & \\
\hline$F 102$ & 14 & $8(9) p / 9(9) m$ & $1.2 / \mathrm{M}$ & $1.2 / 3.4$ & & /3.4 & $/ \mathrm{M}$ & 1.21 & & $/ \mathrm{M}$ & & $1.2 / 3.4$ & OP/OM \\
\hline F1331 & 11 & $9(9) \mathrm{p} / 8(8) \mathrm{m}$ & $1 . / 3.4$ & $1 . / 3.4$ & B. & $1.2 / 3$ & $1 . /$ & $1.2 / 3$ & & 1.4 & & 1.21 & $\mathrm{OP} / \mathrm{OM}$ \\
\hline F1332 & 10 & $4(6) \mathrm{p} / 7(11) \mathrm{m}$ & $1 . / 3.4$ & $1.2 / 3$ & B. & & /3.4 & & & & & $1 . / .4$ & OP/OM \\
\hline F1347 & 10 & $8(10) \mathrm{p} / 5(9) \mathrm{m}$ & 1./.4 & $1.2 / 3.4$ & & $1 . / 3$. & .21 & & & 1./ & & $1.2 / .4$ & OP/OM \\
\hline F1362 & 11 & $6(8) \mathrm{p} / 9(10) \mathrm{m}$ & $1.2 / 3$ & $.2 / .4$ & & $1 . / 3.4$ & & 1.4 & & 1./3. & 13. & $.2 / 3.4$ & OP/OM \\
\hline F1413 & 15 & $11(11) \mathrm{p} / 10(11) \mathrm{m}$ & $1.2 / 3.4$ & $1.2 / 3.4$ & $1 . / 3.4$ & $1.2 / 3.4$ & & 1.21 & 13. & & & $1.2 / .4$ & $\mathrm{OP} / \mathrm{OM}$ \\
\hline F1416 & 9 & $7(10) \mathrm{p} / 7(9) \mathrm{m}$ & $.2 / .4$ & $1.2 / 3.4$ & & $1.2 / 3$ & & & & 1.4 & & $1.2 / 3.4$ & OP/OM \\
\hline F884 & 12 & $10(11) \mathrm{p} / 7(8) \mathrm{m}$ & $1.2 / 3$. & $1.2 / 3$. & .21 & $1.2 / 3$. & .21 & 13.4 & & & & $1.2 / 3.4$ & $\mathrm{OP} / \mathrm{OM}$ \\
\hline Total (c) & & $63(74) p / 62(74)$ & 12.11 & 14.13 & 2.4 & 10.10 & 3.3 & 6.4 & 0.1 & 2.4 & 0.1 & 14.11 & OP/OM \\
\hline \multirow[t]{2}{*}{ Total (d) } & & $125(148)$ & 23 & 27 & 6 & 20 & 6 & 10 & 1 & 6 & 1 & 25 & 0 \\
\hline & & DNF 24 (pAC 365) & & & & & & & & & & & \\
\hline$F 102$ & 14 & $5(8) \mathrm{p} / 3(5) \mathrm{m}$ & /M & P/ & & /M & & 1.21 & & /M & 1.21 & & $O P / O M$ \\
\hline F1331 & 11 & $6(8) \mathrm{p} / 8(10) \mathrm{m}$ & $\mathrm{P} / 3.4$ & $1 . / 3$. & & $1.2 / 3.4$ & & & & B. & $1.2 / 3$ & & $\mathrm{OP} / 1 \mathrm{M}$ \\
\hline F1332 & 10 & 6(7)p/9(10)m & $/ \mathrm{M}$ & $1.2 / \mathrm{M}$ & B. & 1./3.4. & & & & 1./3.4 & $1.2 / 3.4$ & & OP/OM \\
\hline F1347 & 10 & $4(6) \mathrm{p} / 5(7) \mathrm{m}$ & /M & B. & 13. & $1.2 / 3.4$ & & & & & & & 2(1)P/OM \\
\hline F1362 & 11 & $6(9) p / 3(6) m$ & $1 . /$ & $\mathrm{P} /$ & & $1.2 / 3.4$ & & & & 1.21 & 1.4 & & $\mathrm{OP} / \mathrm{OM}$ \\
\hline F1413 & 15 & $7(9) \mathrm{p} / 10(12) \mathrm{m}$ & $1.2 / .4$ & $1.2 / 3.4$ & $1 . / 3.4$ & $1.2 / 3.4$ & & & /3. & 1.4 & 13. & & $\mathrm{OP} / \mathrm{OM}$ \\
\hline F1416 & 9 & $4(7) \mathrm{p} / 9(10) \mathrm{m}$ & $\mathrm{P} /$ & $1 . / 3.4$ & & $1.2 / 3.4$ & & & & /M & $/ M$ & & $\mathrm{OP} / 2(1) \mathrm{M}$ \\
\hline F 884 & 12 & 7(8)p/10(10)m & $1.2 / 3$ & B. & & $1.2 / 3$ & & $1.4(\mathrm{e})$ & & 1./M & $1.2 / 3.4$ & & $\mathrm{OP} / \mathrm{OM}$ \\
\hline Total (c) & & $45(62) \mathrm{p} / 56(70) \mathrm{m}$ & 7.7 & 8.8 & 1.4 & 13.14 & 0.0 & 2.4 & 0.1 & 4.7 & 8.8 & 0.0 & $2(1) \mathrm{P} / 3(2) \mathrm{N}$ \\
\hline Total (d) & & 101(132)(e) & 14 & 16 & 5 & 27 & 0 & $6(\mathrm{e})$ & 1 & 11 & 16 & 0 & $5(3)$ \\
\hline
\end{tabular}

Note. For each of the eight families, and for both minisatellites, the chromosomal assignments deduced from segregation analyses are indicated. The number of children available in each family is indicated (column 2). Column 3 indicates the number of informative bands on the Southern and (a) the total number of bands (including noninformative bands). For each site, 1, 2, 3, 4, $\mathrm{m}$ and $\mathrm{p}$ indicate the grandparental origin of the assigned bands: 1, paternal grandfather; 2, paternal grandmother; 3, maternal grandfather; 4, maternal grandmother; $p$, paternal band of unknown origin; $\mathrm{m}$, maternal band of unknown origin (unknown origin usually reflects the presence of a band with identical size in both grandparents; all families except F102 are three-generation families). The rightmost column reports the number and origin of unassigned bands. In some individuals, two bands are unassigned but correspond to a single locus (b). Total (c) reports the total number of bands analyzed, taking into account parental origin. Total (d) cumulates the data. In one instance (DNF24, F 884), four bands coming from the maternal grandmother segregate like 8pter loci (e), suggesting that in this individual proximal region 2 has been amplified fourfold.

deduce the chromosomal distribution of the loci detected by the two minisatellites. The pattern obtained in CEPH (Centre d'Etudes du Polymorphisme Humain) family 1362 is presented in Fig. 1. Eight bands, numbered 1 to 8 , are detected in the father, Id 136201 , and 10 bands numbered 13 to 22 are visible in the maternal lane, Id 1362 02. The chromosomal assignment deduced from two-point linkage analysis against published data available in CEPH database version 8.1, is indicated at the left- and right-hand sides. In family 1362, all the bands can be assigned to a chromosome, except band 8, which is not informative, and bands 6 and 16, which are a superposition of two alleles segregating independently in the progeny. Altogether, 125 segregations can be scored in the eight families using CEB92, all of which are identical to the segregation from at least one CEPH database marker. The cosegregating markers originate from a limited number of loci. In particular the most distal loci on chromosome 1 (p), chromosome 5 (q), chromosome 6 (q), and chromosome 17 (q) account for 95 of the 125 bands. Loci 6pter, 7pter, 8pter, and 11pter segregations account for 28 of the bands. Loci 9qter and 15qter are each suggested by only one segregation (Table 1 ).

Using pAC365 (DNF 24) and following the same procedure, 101 bands can be analyzed. Five (corresponding to three loci) do not correspond to any pattern in the database. Seventy-three of the remaining 96 seg- regations correspond to the most distal locations typed at $1 p, 5 q, 6 q$, and $15 q$. Twenty-two coincide with the most distal informative loci on $6 p, 8 p$, and $11 p$. Distal $9 q$ is suggested by 1 band. The data obtained strongly suggest that the two loci are colocalized and linked on six chromosome ends ( $1 p, 5 q, 6 q, 6 p, 8 p, 11 p)$, with additionally weak evidence for a $9 q$ localization. In contrast, CEB92 (and not pAC365) is detected at a moderate frequency on 7pter and at high frequency at 17qter. Chromosome 15qter is the second most fre quent pAC365 site (16 bands) but is suspected only once with CEB92 (Table 1). It al so suggests that some of these sites are "major" sites on which one or both loci are usually present (1pter, 5qter, 6qter, 15qter, 17qter) and that the others $(6 p, 7 p, 8 p, 9 q, 11 p)$ are more rarely occupied. One explanation for these findings is that a duplication containing both loci is found at a number of chromosome ends, with the exception of chromosome sites $7 p, 15 q$, and $17 q$, where the duplication is shorter for some reason.

Structural Analysis of the Four Major Sites, 1pter, 5qter, 6qter, and 17qter

Cosmid contigs corresponding to the four major CEB92 sites were built in parallel starting from CEB92 by screening chromosome-specific libraries (chromosomes 1, 6, and 17) or cosmid subclones from 


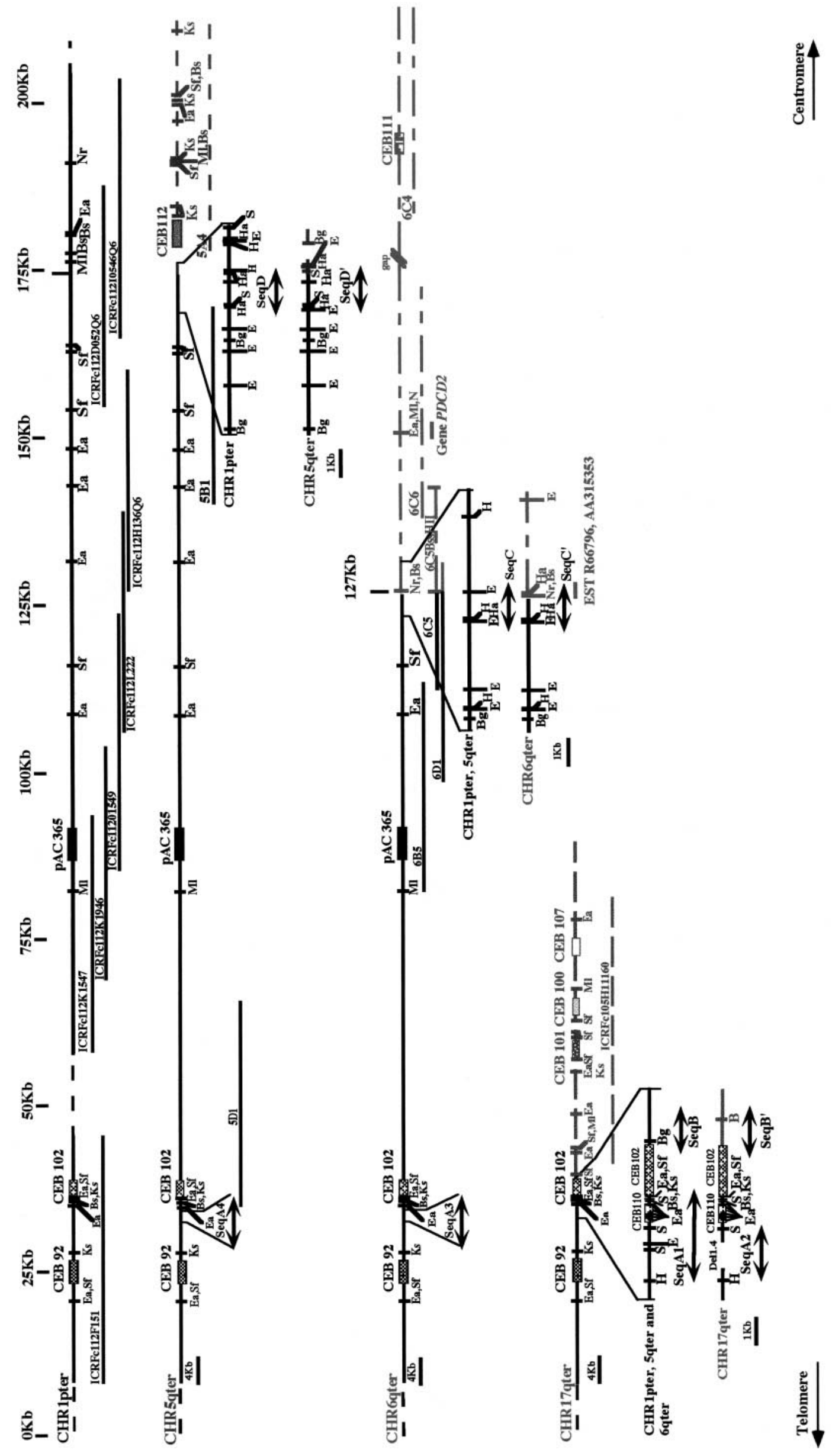


tel omeric half-Y ACs (chromosomes 5 and 6; NIH and Institute of Molecular Medicine Collaboration, 1996). The walk in one direction quickly reached an end as no clone could be obtained from any source after a single step. Restriction maps in the vicinity of CEB92 were identical when taking into account minisatellite allele size differences, suggesting that the different loci are highly homologous. Accordingly the EcoRI restriction map could be used to easily identify and roughly locate breaks in homology of one contig compared to the others. The result of the walks is outlined in Fig. 2. The chromosome 17 restriction map becomes very different from the other three $10 \mathrm{~kb}$ away from CEB92 (defining Region 1). The chromosome 6 restriction map diverges from the chromosome 1-5 maps at $100 \mathrm{~kb}$ from CEB92 (Region 2). Finally, chromosomes 5 and 1 diverge $155 \mathrm{~kb}$ away from CEB92 (Region 3). Polymorphic monolocus minisatellites are located within $30 \mathrm{~kb}$ after the divergence point is reached on chromosomes 17,6 , and 5 , and the segregation data obtained by screening the CEPH families confirm the chromosomal assignment to respectively 17qter, 6qter, and 5qter. The distance between CEB92 and the telomere is approximately $30 \mathrm{~kb}$, as estimated by comparing the size of the 5qter YAC (B22; $225 \mathrm{~kb}$; Kvaloy, 1993) to the distance between CEB92 and the cloning site in B22. This explains the failure to extend the walk in this direction and orients the four contigs with respect to the telomere. CEB92 and pAC365 are $60 \mathrm{~kb}$ apart and pAC365 is absent on chromosome 17, thus explaining the chromosome 17 linkage analysis results.

\section{Structural Boundaries Defined by the Four Major Sites and J unction Sequencing}

J unction $17 q$ versus $1 p, 5 q$, and $6 q$ sites. The region distal to the junction and common to the four sites contains two minisatellite sequences separated by 540 bp and called CEB110 (most distal) and CEB102 (Fig. 2). The translocation occurred within the CEB 102 minisatellite. The chromosome 17 allele contains an integer number of repeats, as deduced from the comparison with the distal end of the minisatellite. The most proximal motif on the chromo- some 5 allele is truncated and the adjacent one is partly deleted (Fig. 3A). A cosmid adjacent to the breakpoint (ICRFC105H 11160, Fig. 2) detects a single locus on human chromosome 17qter by FISH analysis, confirming that the end of the subtel omeric domain has been reached on chromosome 17 (data not shown).

$J$ unction $6 q$ versus $1 p$ and $5 q$ sites. The breakpoint between the 6qter contig and the 1p-5qter contigs is localized $90 \mathrm{~kb}$ proximal in cosmids $6 \mathrm{C} 5$ and $6 \mathrm{D} 1$. The next cosmid, $6 \mathrm{C} 6$ (Fig. 2), detects a single signal on 6 qter by FISH analysis. Three highly polymorphic monolocus minisatellites, CEB111, CEB113, and CEB114 (Cox et al., 1996), are present in cosmid 6C4, which also contains the proximal end of the YAC insert. Comparison between the cosmid restriction maps indicates the position of the translocation breakpoint. The telomeric sides of the $5 q$ ter and 6qter junction are perfectly identical along 80 bp until the breakpoint is reached (Fig. $3 \mathrm{~B})$, approximately in the middle of cosmid 6C5. To investigate whether the proximal half of cosmid 6C5 is chromosome 6 specific or still part of a subtelomeric region, the corresponding fragment as obtained by BsSHII digestion of the cosmid (Fig. 2) was used for in situ investigations. Locus 6qter was detected in $\mathrm{CEPH}$ parents Id136201 and 141301 (Fig. 4), as expected. However, the probe also detected 8pter and 19pter. This demonstrates that the beginning of the chromosome 6-specific domain is not defined, in the present case, by the junction and that the probe defines an additional human subtelomeric layer, subsequently called region 4, which is common to $8 p, 19 p$, and $6 q$ and absent on $1 p$ and $5 \mathrm{q}$.

$J$ unction $5 q$ versus $1 p$. The boundary of the duplicated region on the 5qter locus is located $50 \mathrm{~kb}$ proximal to the chromosome 1-5 vs chromosome 6 divergence point. The junction sequences are aligned from telomere toward centromere in $\mathrm{Fig}$. $3 \mathrm{C}$, showing that the breakpoint is within the poly $(A)$ tract (atypical poly $(A)$ termination: $\left.C(A)_{7} C(A)_{5} C(A)_{5} C A C A\right)$ of an Alu repeat. The sequence CTTTACAG is present at both sides of the Alu repeat on chromosome 5 (underlined boldface on Fig. 3C), whereas no such repetition is found on the 1pter allele, suggesting that the 5qter allele is an ancestor of the 1pter allele. Cosmid 5A4

FIG. 2. Physical map of the four major sites including the junction with the chromosome-specific domain. Restriction map of the translocated region in the loci 1pter, 5qter, 6qter, and 17qter. Solid black lines, translocated region; dotted lines, chromosome-specific domains. These maps are maps of single homologues. Distances from telomere, deduced from the chromosome 5 B22 half-Y AC (K val oy, 1993), may be very different for other homologues due to chromosome end polymorphisms. Restriction map with rare-cutter enzymes Bs, BssHII; $\mathrm{Ea}, \mathrm{Eagl}$; Ks, Kspl; MI, Mlul; N, Notl; Nr, Nrul; and Sf, Sfil and detailed map with current enzymes B, BamHI; Bg, BgllI; E, EcoRI; H, HindlII; and S, Smal. A few HaellI (Ha) sites quoted in the text are also indicated. Cosmids used for in situ hybridization are indicated as solid lines. The $6 q$ ter contig is interrupted between cosmids $6 \mathrm{C} 6$ and $6 \mathrm{C} 4$ by a gap of $60 \mathrm{~kb}$. 1374 nucleotides are absent at the $17 \mathrm{qter}$ locus and are indicated by Del1.4. Minisatellites are drawn as boxes: CEB92 (Accession No. (AC) Y13539), CEB110 (AC Y13547), CEB102 (AC Y 13546, Y 13547, Y 13548), CEB 111 (Ac Y 13538). Other sequenced fragments are indicated by double arrows: Seqs A1 (chromosome 1p; Ac Y 13543), A2 (chromosome 17q; Ac Y 13546), A3 (chromosome 6q; Ac Y 13545), and A4 (chromosome 5q; Ac Y 13544), 2 kb sequenced for evaluation of the divergence (see Table 1); Seqs B (Ac Y 13548) and B' (Ac Y 13540), 1pter-and 17qter-derived sequences overlapping the junction with the 17qter domain reported in Fig. 3A; Seqs C (AcY 13549) and C' (Ac Y 13541), 1p-and 6q-derived sequences overlapping the junction with 6qter domain 4 as reported in Fig. 3B; and Seqs D (Ac Y 13550) and D' (Ac Y 13542), 1p- and 5q-derived sequences overlapping the junction with 5 qter as reported in Fig. 3 C. 
following the junction contains the YAC vector cloning site and is specific to human chromosome 5 as shown by FISH. The chromosome 5qter contig is built from cosmids subcloned from a YAC and was not confirmed by an independent genomic library resource. Theoretically, the deviation from this chromosome's map could be the result of a cloning artifact in the half-YAC. This is unlikely because of the FISH data. More importantly, an artifact junction would not easily create the short duplication flanking the chromosome $5 \mathrm{Alu}$ se quence and present only once at the chromosome 1 junction (Fig. 3C).

\section{Sequence Similarity between the Four Major Sites}

The EcoRI and rare-cutter restriction maps of the contigs are almost identical in the region of overlap, and the limited sequence analysis done at the distal side of the junction points further suggests that the different sites are highly similar.

To precisely measure the sequence similarity between the four different sites, the sequence corresponding to the $2 \mathrm{~kb}$ just distal to CEB110 (Fig. 2) was determined for one chromosome 1 and two chromosome 5, 6, and 17 independent alleles (Accession Nos. Y 13542 to Y 13547; more detailed information can also be found at our site, http://www.igmors.u-psud.fr/iech). The sequence on the chromosome 17 alleles is only 639 bp long due to the absence of 1374 nucleotides. The divergence between the heterologous chromosome sites is approximately $1 \%(0.56-1.44 \%$ for $2034 \mathrm{bp}$ and $0.2-$ $1.8 \%$ for $639 \mathrm{bp}, \mathrm{P}<0.05)$ and is not significantly higher than the divergence between the homologous alleles, suggesting a divergence time of not more than 1-2 million years for the major sites (Efstratiadis et al., 1980).

\section{Analysis of Putative CpG Islands}

CpG islands appear to be good indicators of the presence of genes in telomeric (GC rich) domains as shown by Flint et al. (1997b). CpG islands are often revealed by the aggregation of recognition sites for the rare cutter enzymes Notl, Eagl, BssHII, Kspl, Mlul, and Nrul. The mapping of the corresponding sites across the duplicated domains reveals two such aggregates. The first is localized $8 \mathrm{~kb}$ proximal to CEB92 (Fig. 2) and comprises Eagl, BssHII, and Kspl sites. The second aggregate is the coincidence of a Nrul and BssHII site in Region 4 immediately proximal to the chromosome 6/chromosomes 1 and 5 junction.

To investigate the nature of the first potential CpG island, a 4-kb fragment was sequenced from a chromosome 5 allele. The proximal half of the 4-kb fragment contains the two minisatel lite tandem repeats CE B110 and CEB102 (Seq A1, Fig. 2). The repeat unit in CEB110 is 69 nucleotides long and contains one Eagl site, thus creating a cluster of Eagl sites over a short distance. The CEB 102 repeat unit is 105 bp long (Fig. $3 \mathrm{~A})$ and the sequenced fragment contains only the dis- tal end of the tandem array. The 540 bp separating CEB110 and the more proximal CEB102 contain the Eagl, BssHII, and Kspl rare-cutter sites. This segment is very GC rich (68\% GC) but with a low (0.54) observed/expected CpG dinucleotide ratio. No significant homology of this fragment with CpG islands or EST sequences could be found.

A sequence database search identifies a strong homology with EST AA315353 in the distal half of the $4-\mathrm{kb}$ fragment that was sequenced on different alleles to measure the site similarities. More precisely, se quence from 265 to 367 of the EST sequence is flanked in the genomic sequence by consensus splicing sites. The homology is within the 1374-bp region absent on chromosome 17. The 103-nucleotide homology is perfect with alleles from 1pter, 5qter, and 6qter. No other part of EST AA315353 is seen in the 4-kb sequence generated and no other significant hit was obtained in the databases.

The BssHII site in the second candidate CpG island is 160 bp proximal to the chromosome 6/chromosomes 1 and 5 junction, and the Nrul site is $60 \mathrm{bp}$ proximal to the BssHII site (Figs. 2 and 3B). A perfect sequence identity with EST AA315353 starts 14 nucleotides after the breakpoint on $6 q$ and runs for 100 nucleotides. I dentity between the three sequences stops abruptly at a consensus splice site on the cosmid (Fig. 3B). The rare-cutter enzyme sites of BssHII and Nrul (Fig. 2) are localized in this intron.

In contrast with the situation observed within the duplication, aggregates of rare-cutter sites are quickly encountered outside of the domain on chromosomes 5 and 6 at least. A Notl site is located on chromosome 6 $25 \mathrm{~kb}$ proximal to the chromosome $6 / 1$ and 5 junction, close to Eagl and Mlul sites (Fig. 2). The sequence of the proximal side of the Notl site starts at the Notl site at position 190 in coding sequence S78085 (PDCD2 gene) and stops abruptly at position 312. The genomic sequence at this position is the good consensus donor splice site GAG/gtgag. PDCD2 was independently assigned to 6q27, with no pseudogene (Kawakami et al., 1995), showing that it is part of the chromosome 6-spe cific domain and probably the very first gene on chromosome $6 \mathrm{q} 150 \mathrm{~kb}$ from the telomere.

\section{In Situ Investigation in CEPH Reference Individuals \\ Id 1362-01, 1413-01, and 1413-02}

To investigate the situation at the other sites suggested by linkage analysis, and to identify the ancestral site, we have undertaken a number of FISH investigations in $\mathrm{CEPH}$ reference individuals and in one chimpanzee and one orangutan cell line.

The three junctions define three subtel omeric layers, Region 1 (telomeric to the 17q junction), Region 2 (be tween the $17 q$ and the $6 q / 5 q-1 p$ junction), and Region 3 (between the $6 q$ and the $5 q / 1 p$ junction). In addition, although not as well defined, a fourth subtelomeric domain is found between the chromosome 1-5/6 junc- 
tion and the chromosome 6-specific domain. The size of Region 1 is estimated at $40 \mathrm{~kb}$, Region 2, $90 \mathrm{~kb}$, and Region 3, $60 \mathrm{~kb}$. Region 4 is less than $25 \mathrm{~kb}$ long and is represented by the proximal half of cosmid 6C5 (see left-hand side in Fig. 4).

As expected, cosmids from Region 1 detect multiple loci in the human cell lines. The results obtained for Id 1362-01 are summarized in Fig. 4A. Region 1 is detected on both homologues at 1pter, 17qter, 6qter, 5qter, 7pter (but with a weaker signal at this site), and 8pter and on one homologue at 11pter and 15qter. Polymorphism for Region 1-associated sites is demonstrated by the absence of signal at 11pter or 15qter and the presence of a signal on both homologues at 6pter, 19pter, and 19qter in Id 141301 (Fig. 4C). Except for 19pter and 19qter, all the sites detected by Region 1 cosmids were suggested by linkage analysis. On a per individual basis, however, linkage analysis expectedly misses some sites (such as 7pter, 8pter, and 15qter in Id 1362-01), presumably because some of the bands detected on the Southern blots are not informative (Fig. 1 and Table 1). As expected, cosmids from Region 2 do not detect 17qter. Cosmids from proximal region 2 containing minisatellite pAC365 (Fig. 2) fail to detect 7pter in all three CEPH individuals, in agreement with linkage data (Table 1), suggesting that 7pter contains approximately the distal half of Region 2. Similarly, the sudden loss of signal on $15 q$ suggests that the chromosome $15 q$ translocation junction is at most 20 $\mathrm{kb}$ distal from the 6qter/1p-5qter junction in Region 2.

Cosmids from Region 3 do not detect 8pter, suggesting that the breakpoint in homology on $8 p$ is very close to the $6 q$ junction. They stop detecting 6pter (observed in Id 1413-02, Fig. 4D) approximately $30 \mathrm{~kb}$ distal to the $5 q-1 p$ junction.

The probe defining Region 4 detects 6qter, 8pter, and 19pter in both Id 136201 and 141301, which confirms the similarity of the $6 q$ and $8 p$ alleles. Region 4 is present on chromosome 19p in Id 136201 despite the fact that Region 1 is absent at this site in this individual (Figs. 4B and 4C).

Region 1 detects both chromosome 9qter homologues in individual 141302, in agreement with linkage data, but with a weaker signal (a behavior similar to 7pter). However, proximal Region 2, which contains minisatellite pAC 365, is not detected in this individual by in situ, in disagreement with linkage data. The reason for this discrepancy is unknown and could reflect additional rearrangements at this site. The in situ investigation in this restricted set of individuals demonstrates the existence of at least three different chromosome 9qter alleles.

\section{In Situ Investigation in Chimpanze and Orangutan}

Region 1 cosmids are monolocus at chromosome 17qter in both chimpanzee and orangutan, and Region 2 is absent at chromosome 17qter in both primates, which is identical to the situation in human.
Cosmids from Regions 2 and 3 detect a number of loci, both telomeric and interstitial, in chimpanzee (Fig. 4D). The interstitial sites, 1q42, 4q27, 7p11, and 7q11 (human nomenclature), are also detected in humans, and some of the telomeric sites are common. Cosmids proximal to the junction on chromosomes 17 and 6 are monolocus and at the same location in the two primates. In contrast, cosmid 5A4, adjacent to the chromosome 5-1 junction on human chromosome 5, and monolocus at 5qter in humans and orangutan, is present at seven chromosome ends (1pter, 3qter, 4qter, 5qter, 8qter, 10pter, and qter) and at an interstitial site (9cen) in chimpanzee (Fig. 4D).

\section{DISCUSSION}

By combining the use of segregation analyses in large families, chromosome walking, and in situ hybridization studies, we have characterized different layers constituting one example of subtel omeric duplications that is of interest in understanding the evolution of chromosome ends in higher primates. A number of specific features made this analysis possible. First, the translocation domain contains two very highly polymorphic minisatellites with a wide allele size range perfectly suited for Southern blot analyses (Fig. 1). This finding has facilitated the identification of the different sites and provided information complementing and strengthening the FISH and physical mapping investigations. Second, although it occurred very re cently, as demonstrated by the extremely close se quence similarity of the different sites analyzed, the domain quickly spread to a significant number of chromosome ends. It has been possible in this study to identify this spreading clearly because this family of duplication events is capped by Region 1. Region 1 is monolocus on chromosome 17qter in both chimpanzee and orangutan, and it is most likely that this site is the ancestral site. This is further suggested by the structure of the Region 1/Region 2 junction. The chromosome 17 CEB102 minisatel lite allele contains a perfect number of repeats, which is a feature common to $70 \%$ of human minisatellites, and both flanking sequences, the telomeric and centromeric side, share with the repeat units a high density of TG motifs (Fig. 3A). On the contrary, alleles at the other sites contain an imperfect number of motifs, the last motif is truncated, and the previous one contains an internal deletion. The two chromosome 17 alleles analyzed lack a 1374-bp fragment that is present on chromosome 1, 5, and 6 alleles and contains one exon of EST AA315353, another part of which is located $90 \mathrm{~kb}$ downstream on the chromosome 6 locus close to the second translocation junction within Region 4. Such split matches appear to be common events in the vicinity of telomeres ( $\mathrm{Nicholls}$ et al., 1987; Flint et al., 1997a), and a third part of EST AA315353 is also found 10 bp proximal to the chromosome 4qter degenerate TTAGGG at the distal/proximal subdomain boundary. 


\section{A}

\section{Telomeric side}

1pter ccagggatgcacgcagagtaaggatgtgtgtgtctacgcatgtgggggt

17 ter ccagggatgcgcgcagagtaaggatgtgtgtgtctacgnatgtgggggt

1pter TGGGTNTGACGGGTGTGTTCTGTGTGAGAACGTGTGTGTAGTGTTCACATGTCCTCTGTGCGTGAGTCCCTGTGTGTGATGTTGTGTTCTCGGTGTGAGTCA

17 gter TNGGNGTACGGGTGTGTTCTGTGT...

1pter TGGGTGTGACGGGTTGTCTGTGTGTGAGAACGTGTGTGTAGTGTCCACATGTCCTCTGTGCGTGAGTCCCTGTGTGTGATGTTGTGTTCTCGGTGTGAGTTCA

1pter TGGGTGTGA.......

1pter TGGGTGTGACGGGGTGTGCTGTGTGAGAGAACATGTGTGTAGTGTTCACATGTCCTCTGTGCGTGAGTCCCTGTGTGTGATGTTGTGTTCTCGGTGTGAGTCA 1pter TGGGTGTGACGGGGTGTGCTGTGTGAGAGAACATGTGTGTAGTGTTCACATGTCCTCTGTGCGTGAGTCCCTATGTGTGATGTTGTGTTCTCAGTGTGAGTTCA

lpter TATGTGTGACGGGTGTGCTGTGTGAGAGAACGTGTTGTAGTGTTCATATGTTCTCAGTGTGTGAG<------------deletion----------->TTCA lpter TGTGTGTGACGGGTGTGCTGTGTGA--GAACCCGTGTGCAGTG

lpter agatcttccccaaaggtagttcaaagctgggccetttcatttcccggatctaaccc

17qter TGGGTGTGACGGGGTGTGCTGTGTGAGAGAACATGTGTGTAGTGTCCACATGNCCTCTGTGCGTGAGTCCCTGTGTGTGATGCCGTGTTCTCAGTGTGAGTTCA

17 ter tgtatgtgtgtagtgagtttatgctcttgtgtgtcagcacatgggtatgatgtgggtatgttctctctgtgggtg

Centromeric side

B

Telor ide

5qter 'CACAATATTTGCCAAAGTCAAGTATCCATTACACTATTAATTTTCATTCTT

6qter AAAAATTCACAATATTTGCCAAAGTCAAGTTATCCATTACACTATTAATTTGTCATTCTT PJ62

5qter TTGTTTATATAGTCAATATCTCTATCTCAATTGGATCTATCTCAACTGCTTCTAAACAAG

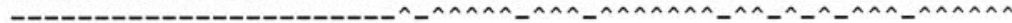

6qter TTGTTTATATAGTCAATATCTCTGTTGACCTGCCAGTGTCTCCGCCGGTTGAAAGCGCGT

EST1 (R66796) 5 'end GTCTCCGCCGGTTGAAAGCGCGT EST2 (AA315353) 5 'end CTCCGCCGGTTGAAAGCGCGT

180

5qter CCACCATAGTCTCTCCCATTTCAACAATCTCTTCCAAGTACCACTTCATTTCTTCTTTTC

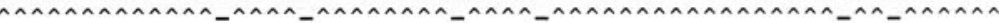

6qter GTCTGCGTCGGGTTCTGTTGGAGTGCGTTCGGTGTGCCGTGGGTC-GCGCTGCTTCCACC PJ61

$---1--1-1-$

EST1 GTCTGCGTCGGGTT

"TGGAGTGCGTTCGGTGTGCCGTGGGTCCGCGCTGCTTCCACC

EST2 GTTTGCNTCGGGTTCTGTTGGAGTGCGTTCGGTGTGCCGTGGGTCCGCGCTGCTTCCACC

5qter ATATTTTTGAAAACTTTTGAAAAACTACCTATTTTCCTCCTCCATTTCTTGTTCATTCC

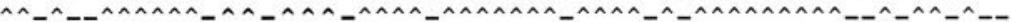

6qter CAACTTCCTGTTAGGTAAGAGGCGCGTGAGGCTCCTGTGCCGGGGGGGTGCTGCTCCC

EST1 CAACTTCCTGTTAGGACCCACCCGAGGACGGTCACCATGGCTTTGGAGCACCTGGTAG

EST2 CAACTTCCTGTTAGCTGCAGGACAAGTTCGAGCATCTTAAAATGATTCAACAGGAGGAG BSSHII

300

5qter ATTCTAGTGGACATGGAATCTGTTCCTCCTCCAAAACGGAATTGGTCACCCTTAAATTA

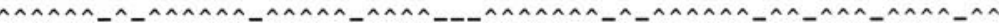

6qter GAGTCGGCGCGCGGCGGGGACGCGAGTCCGTAGGTGCTGGCGGGAGCGAGAGTCGGGTGG

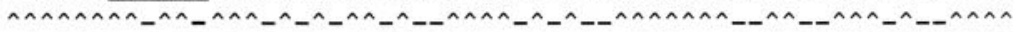

EST1 TGTGGCATGTCCACAGTGAAGACCAGAGTTTCGTTGTCCTTAAGACTGACCTGGGGAGAC

EST2 ATAAGGAAGCTCGAGGAAGAGAAAAAACAACTGGAAGGAGAAATCATAGATTTTTATAAA NruI HaeIII

5qter CTAAACCCAAAACAATATGTTGTCTTTATCTTTACCTCTCTGTGGCATTTAATGATAAGA

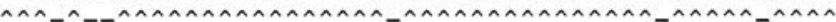

6qter GGGACCCTCGCGAGCCCGCACTCCGCCTCTGGGTAGCAGCCTCTTCGGCC

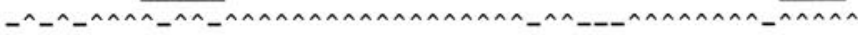

EST1 GTGGCTGCAGGCATTTGAGGAAGACGGCACCGAAAGCGAAGGAAGCTCCTGCTCCTCCTA

EST2 ATGAAAGCTGCCTCTGAAGCACTGCAGACTCAGCTGAGCACCGATACAAAGAAAGACAAA 399

ECORI

5qpter CCACTACTTTCTTCTCTTTTACCCTTCTTTCTTGAATTC

EST1 AAGCCGAAGCCAAAGTGAAGGCTTTAAAGGCCAAGAAGGCAGTGTTTGAAAGGTGTTCCG

EST2 CATCCTCTGCTGCCCCCTGCCCCATACACGTGATGGAGCAGAAAACGTGCTGTGTGAACC

EST2 TGTGACTTCAGGGCCTGTTGACGTGGTCGTGCTTGCATACTCTCTGG 
C

Telomeric side 1

1pter GAAATAAGACTTTACAGCAGCCGGGTGCAGTGGTGCAGGCCTGTAATCCCAGCACTTTGG

5qter GAAATAAGACTTTACAGCAGCCGGGTGAGTGGTGAGGCCTGTAATCCCAGCACTTTGG HSALUCOL $\hat{\text { GGCCGGCGCGGTGGTCAC GC CTGTAATCTCAGCACTTTGG }}$

HaeIII

120

1pter CA-GCAGAGGCAGGCGGATCGCTTTGAGCTCAGGGCAACATAGCCAAAACCCCCCTCCCT

5 ter CA-GCAGAGGCAGGCGGATCACTTTGAGCTCAGGGCAACATAGCCAAAAGCCCCCTCCNT

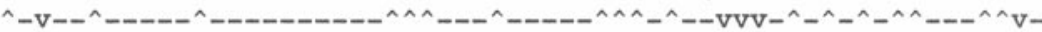

(Alu) GAGGCCGAGGCGGGCGGATCAC---GAGGTCAGG---AGAT---CGAGACCATCCTGGCT

180

1pter AGNNNACCCCCACCCCCTCCCTACCAAAAATACAAAA---CAGCAGGGCATGGTGGCGGG

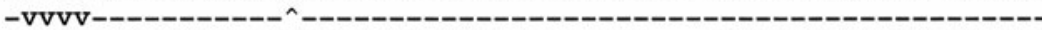

5 ter ANNNNACCCCCACCCCGTCCCTACCAAAAATACAAAA---CAGCAGGGCATGGTGGCGGG

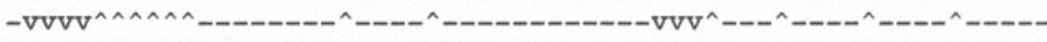

(Alu) AACACGGTGAACCCCGTCTCTACTAAAAATACAAAAAATTAGCCGGGCGTGGTAGCGGG

240

1pter CGCCTGGTAGTCCCAGCTACTCAGGAGGCTGAGGCAGGAGAATCACCTGAACCCAGGAGG

5qter CGCCTGGTAGTCCAAGTACTCAGGAGGTGAGGAGGAGAATCACCTGAACCCAGGAGG

Alu) CGCCT-GTAGTCCCAGCTACTCGGGAGGCTGAGGCAGGAGAATGGCGTAACCCGGGAGG

300

1pter CAGACATTGCAGTGAGCCAAGATCACGCCACTG----CCAGCCTGGATGACAGAGCAAGA

5qter CAGACATTGCAGTGAGCCAAGATCACGCCACTG----CCAGCCTGGATGACAGAGCAAGA

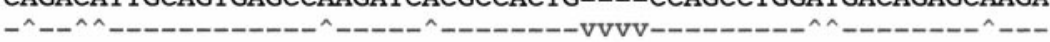

(Alu) CGGAGCTTGCAGTGAGCCGAGATCGCCCACTGCACTCCAGCCTGGGCGACAGAGCGAGA

345

*hscb7 CAAAAAAACAAAAACAAAAA--CAAA--TCAGAATGGGA

lpter CTCCACCTCAAAAAAACAAAAACAAAAACACAAACCTCAGAGCAC

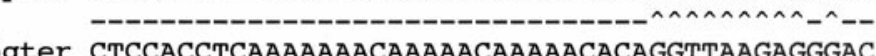

5qter CTCCACCTCAAAAAAACAAAAACAAAAACACAGGTTAAGAGGGAC

(Alu) CTCCGTCTCAAAAAAAAAAAAAAAAAA

1pter CCCCAGGTATTCCAACCTAATCCTGGTGCCCCGCСTCTCACCACCСTTCTTCCTGTTTAA

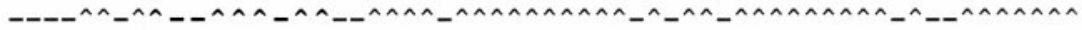

5 ter CCCCGAGCCTTTACAGATACAAGTTTAAGAGGACCTCTAAGCAAAAAATGCCAACCCTT

Centromeric side

FIG. 3-Continued

It has been suggested (Flint et al., 1997a) that the TTAGGG motifs and that distal and proximal subdosubtelomeric domain is separated into a distal and a mains are not transferred together in a translocation proximal part by a short stretch of degenerate event. In agreement with this view, the chromosomal

FIG. 3. Sequence of the junction points between the four sites. (A) Sequence of the junction between 17qter and (1pter, 5qter, and 6qter). Seqs B (Ac Y 13548) and B' (Ac Y 13540; see Fig. 2) are aligned from their telomeric ends. The common region is in black, the 17qter-specific area is gray. The telomeric flanking sequence of the minisatellite CEB102 is common to 1pter/17qter. Lowercase letters indicate sequence flanking the tandem repeat array. Repeat motifs are uppercased. Variant nucleotides between the repeats are gray. The BgllI (bold, italicized) restriction site in the immediate chromosome 1pter flanking sequence has been localized in the detailed map of Fig. 2. The last unit at the 1pter minisatel lite allele is shortest and is 44 nucleotides long instead of 105 nucleotides for a normal repeat. The minisatellite at the 17qter allele ends with a normal size repeat. The 17qter centromeric flanking sequence is very rich in TG motifs, reminiscent of the tel omeric side and of the minisatellite repeat unit. (B) Sequence of the junction between the 6qter contig and the (1pter and 5qter) contigs. The sequences from 5qter (Seq C, see Fig. 2) and from 6qter (Seq C', see Fig. 2) are aligned from the telomeric end. Sequences from the translocated domain (in black), 6qter-specific sequences (in gray), and EST1 (R66796) and EST2 (AA315353) (in gray). The consensus splice site at position 200 (bold, italicized) marks the end of the sequence identity between the ESTs and chromosome 6qter. Restriction sites indicated in the detailed map of Fig. 2 are italicized and underlined. Primers PJ 61 and PJ 62 have been used to sequence the junction point from one 1pter (ICRFC112L 222) and one 6qter (ICRF C109C0311Q5) ICRF cosmid. The underlined sequence of EST AA315353 is found on Region 1 upstream of minisatellite CEB110 on chromosomes 1, 5, and 6 but is absent from chromosome 17. (C) Sequence of the junction between 1pter and 5qter loci. Sequences D and D' (see Fig. 2) are aligned together with the most homologous Alu sequence found in the REPBASE collection of repeated elements: HSALUCOL. The Alu sequence is in gray. The direct repeats flanking the Alu element in the 5qter sequence are boldface and underlined. *Alu element HSCB79B 4 has a 3' end homologous to the 1pter sequence, suggesting that the 1pter locus might have contained an Alu element before the translocation occurred (i.e., inter-Alu-element recombination event). 


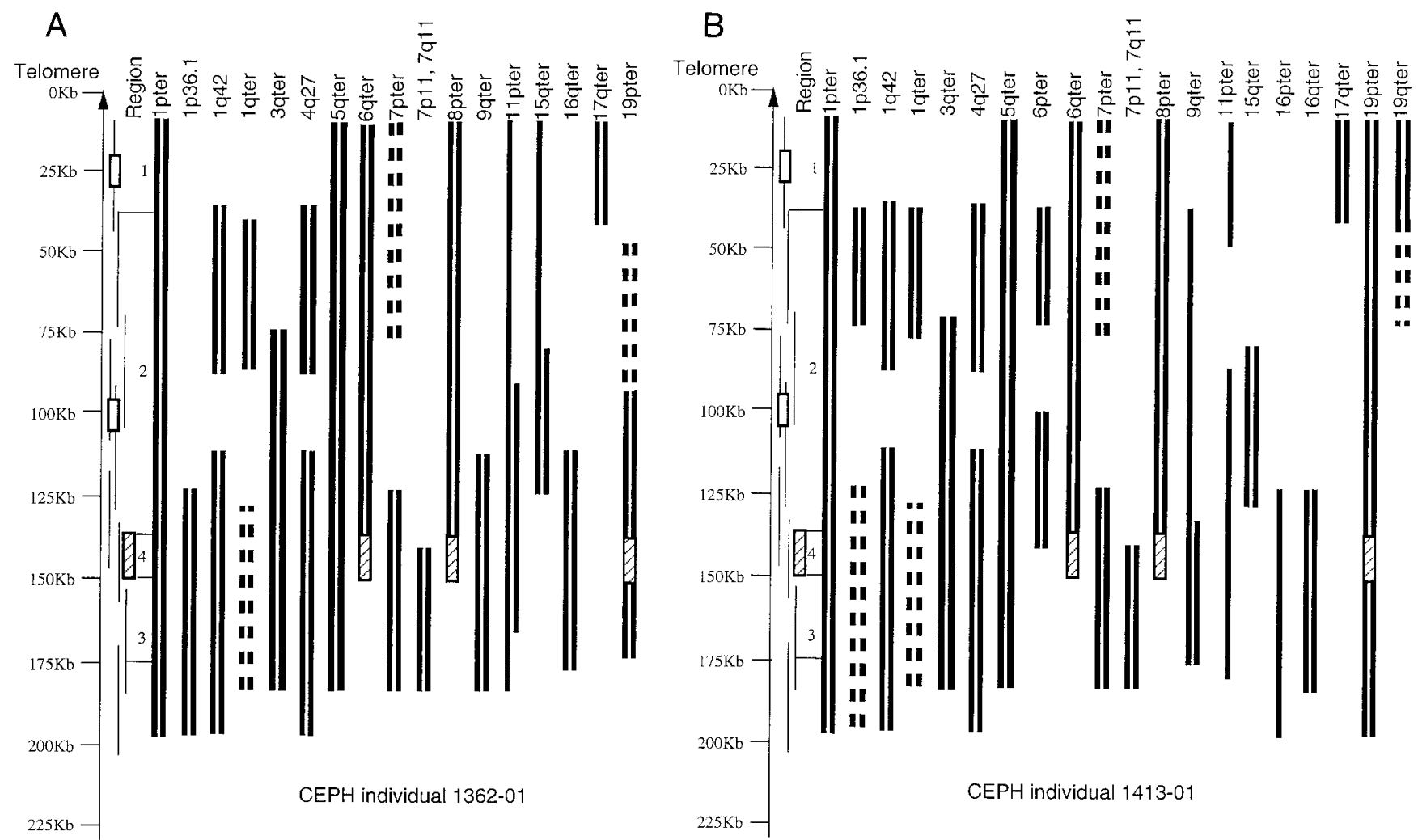

FIG. 4. Summary of the in situ investigations. Cell lines from CEPH parents 136201, 141301, and 141302 and from a chimpanzee were investigated in situ using cosmids ICRFC112F151 (Region 1), 5D1, ICRFC112K1547, ICRFc112K 1946, ICRFc11201549 (Region 2), ICRFC112L222 (Regions 2 and 3), ICRFC112H136Q6, ICRFc112D052Q6, and ICRFc112I0546Q6 (Region 3) (Fig. 2). The hatched box represents the signal of subclone "6C5BsshII," shown in Fig. 2 and defining Region 4 . Solid bars represent a supposedly continuous signal of overlapping cosmids for one chromosome. Interrupted lines (7pter, 9qter) represent weaker signals. Ten metaphases were observed for each cosmid. (A) Id 1362-01, (B) Id 1413-01, and (C) I d 1413-02.(D) Sites identified in chimpanzee (chromosome numbers refer to the numbers of the human homologues). The gray bars represent the signal obtained with cosmid 5A4, adjacent to the chromosome $5 / 1$ junction, and monolocus at 5qter in both human and orangutan. Orangutan was investigated with Region 1 and Region 3 cosmids. Region 1 is monolocus at 17qter; Region 3 is observed at 3qter, 7p11, 7q11, and 7qter. The two cosmids adjacent to the junction at 17qter and 6qter are monolocus at the corresponding site in orangutan and chimpanzee.

distribution of the distal subdomain from the 6qter half-YAC yRM2158 as deduced from Macina et al. (1995) is different from the chromosomal distribution of the proximal part as described in the present study. Similarly, the telomere-associated sequence TelBam11 (Brown et al., 1990) is detected at many 17qter and 5 qter alleles but not at 1pter or 6qter sites. However, it should be stressed that even a limited number of unequal subtelomeric translocations occurring at a low frequency during our genome evolution can produce a very intricate pattern of low-copy repeat regions. Regions 2, 3, and 4 are present, together or separately, at many other chromosome ends, in both human and chimpanzee. Some of the sites detected are interstitial, presumably reflecting events (such as chromosome end fusion) that occurred in a common ancestor. Data generated in different laboratories cannot be compared and discussed unless common, widely accessible reference individuals are used, such as CEPH parents 1362-01 and 1413-01, who correspond to two of the largest and most studied families in the making of human genetic maps. It would be even more signifi- cant, obviously, regarding studies aimed at unraveling the recent evolution of our chromosome ends and comparing distal and proximal subtelomeric domains, to use a collection of reference cell lines from different well-defined human populations. The reconstructed history of the spreading of Region 1 , by dedicated studies using such resources, will have to be compatible with a number of observations made here. For instance, one possible sequence of events could be 17qter $\rightarrow$ 7pter getting distal Region $2 \rightarrow$ 15qter getting proximal Region $2 \rightarrow$ 19pter getting Region 4 and cre ating the split match $\rightarrow 6$ qter; 5 qter $\rightarrow$ 1pter. The most likely mechanism for the spreading is the presence of a cryptic balanced translocation in one parent followed by an unbalanced transmission to some of the progeny. This proposed succession of events makes the easily testable prediction, in comparison with the results re garding 7pter, presented in Trask et al. (1998), that Region 1 distribution is strikingly different in Pygmy populations (and perhaps even monolocus at 17qter).

Indeed the evolutionary significance of these translocations remains undear. The definition of the subte- 

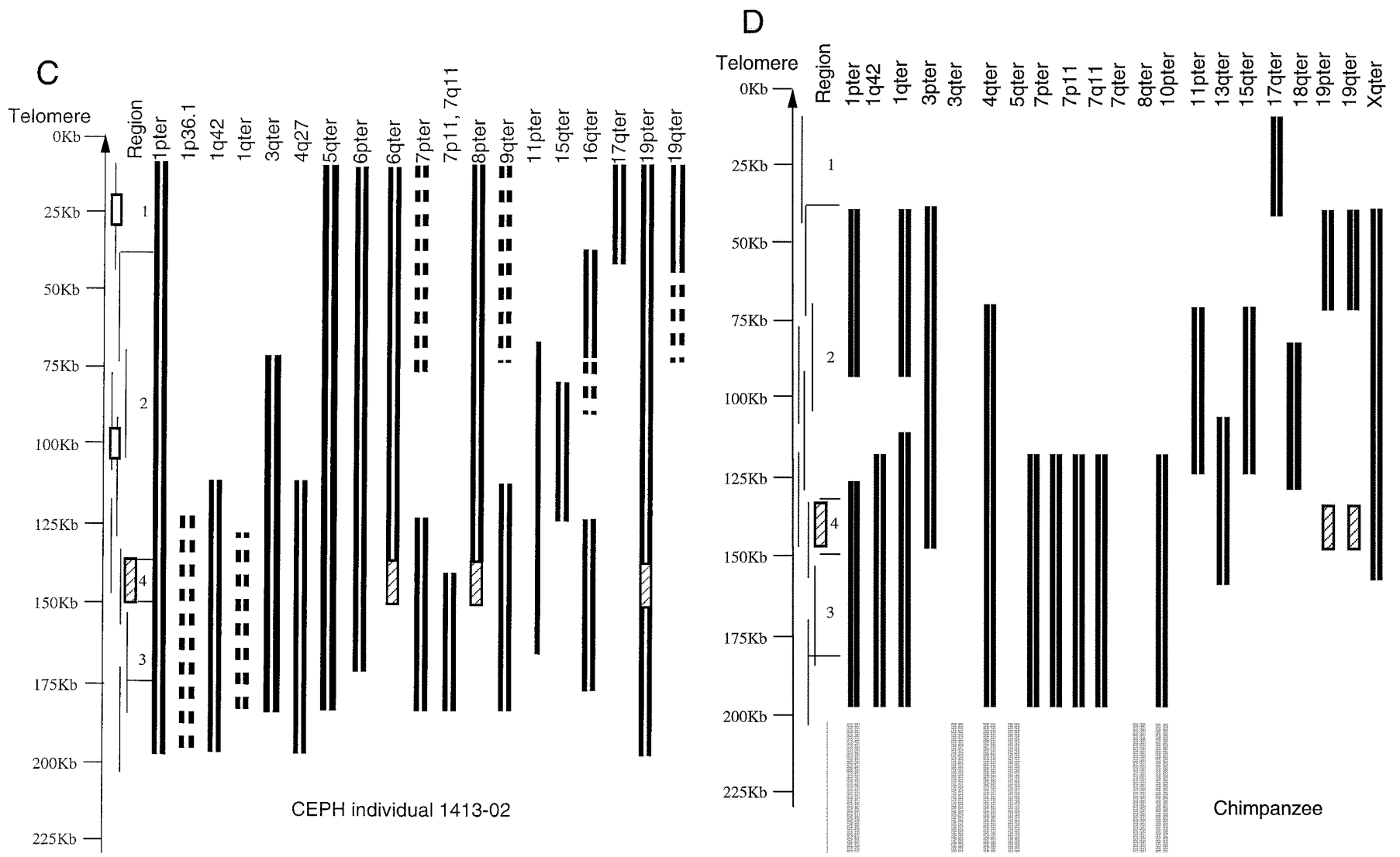

FIG. 4-Continued

lomeric domain as the area between the tel omere itself and the start of chromosome-specific sequence is relative. Region 1 is monolocus in the two primates studied and not in human, whereas cosmid 5A4 is monolocus in both human and orangutan but not in chimpanzee. The very rapid establishment of new proximal subtel omeric domains could suggest that the domain confers some sel ective advantage. Chromosome 6qter is of particular interest because PDCD2 (Kawakami et al., 1995), a highly conserved gene, is located just proximal to the translocation. Riethman and colleagues (Macina et al., 1995) identified by RARE cleavage one 6qter allele 130 $\mathrm{kb}$ larger than the others in a collection of 11 individuals and ther efore demonstrated that 6qter is polymorphic in the human population. We predict that this rare allele is the chromosome 6qter ancestor, devoid of Region 4. This, in association with the search for phenotypes associated with homozygosity for the rare allele, may provide an efficient tool for the study of the functional significance of subtel omeric domains.

\section{ACKNO WLEDGMENTS}

We thank Professors J ean Dausset and Howard Cann for the provision of DNA samples from the CEPH reference panel and of cell lines from selected individuals. We are very grateful to William Brown and Harold Riethman for providing telomeric YACs for re spectively 5qter and 6qter and to J onathan Flint for providing cos- mid subclones from these YACs. We thank Mourad Sahbatou at CEPH for a final two-point lod score analysis of segregation data against $\mathrm{CEPH}$ ver8.1 data. More detailed data can be found at http://igmors.u-psud.fr/iech. This work was supported by grants from the EEC (EUROGEM Project EC Contract GENE-CT93-0101), from the French Ministry of Research (ACC-SV grant), and from the GIP-GREG. S.M. has been supported by a stipend from the Ministère de la Recherche and from the Association Française contre les Myopathies.

\section{REFERENCES}

Baird, D. M., and Royle, N. J . (1997). Sequences from higher primates orthologous to the human $\mathrm{Xp} / \mathrm{Yp}$ telomere junction region reveal gross rearrangements and high levels of divergence. Hum. Mol. Genet. 6: 2291-2299.

Brown, W. R. A., Mackinnon, P. J ., Villasanté, A., Spurr, N., Buckle, V., and Dobson, M. J. (1990). Structure and polymorphism of human telomere-associated DNA. Cell 63: 119-132.

Cox, S. A., Attwood, J ., Bryant, S. P., Bains, R., Povey, S., Rebello, M., Kapsetaki, M., Moschonas, N. K., Grzeschik, K. H., Otto, M., Dixon, M., Sudworth, H. E., Kooy, R. F., Wright, A., Teague, P., Terrenato, L., Vergnaud, G., Monfouilloux, S., Weissenbach, J ., Alibert, O., Dib, C., Fauré, S., Bakker, E., Pearson, N. M., Vossen, R. H. A. M., Gal, A., Mueller-Myhsok, B., Cann, H. M., and Spurr, N. K. (1996). European Gene Mapping Project (EUROGEM): Breakpoint panels for human chromosomes based on the $\mathrm{CEPH}$ reference families. Ann. Hum. Genet. 447- 486.

Efstratiadis, A., Posakony, J. W., Maniatis, T., Lawn, R. M., O'Connel, C., Spritz, R. A., DeRiel, J . K., Forget, B. G., Weissman, S. M., Slightom, J . L., Blechl, A. E., Smithies, O., Baralle, F. E., 
Shoulders, C. C., and Proudfoot, N. J . (1980). The structure and evolution of the human beta-globin gene family. Cell 21: 653- 668.

Flint, J ., Bates, G. P., Clark, K., Dorman, A., Willinghan, D., Roe, B. A., Micklem, G., Higgs, D. R., and Louis, E. J . (1997a). Sequence comparison of human and yeast telomeres identifies structurally distinct subtelomeric domains. Hum. Mol. Genet. 6: 1305-1314.

Flint, J ., Thomas, K., Micklem, G., Raynham, H., Clark, K., Doggett, N. A., King, A., and Higgs, D. R. (1997b). The relationship between chromosome structure and function at a human telomeric region. Nat. Genet. 15: 252-257.

Green, P., Falls, K., and Crooks, S. (1990). Documentation for CRIMAP, version 2.4. Washington University School of Medicine, St. Louis, MO.

Ip, N. Y., Nicholas, L., Baum, H., and Balazs, I. (1989). Discovery of a novel multilocus DNA polymorphism [DNF24]. Nucleic Acids Res. 17: 4427.

Kawakami, T., Furukawa, Y., Sudo, K., Saito, H., Takami, S., Takahashi, E., and Nakamura, Y. (1995). I solation and mapping of a human gene (PDCD2) that is highly homologous to Rp8, a rat gene associated with programmed cell death. Cytogenet. Cell. Genet. 71: 41- 43.

Kvaloy, K. (1993). Ph.D. thesis, Oxford University.

Macina, R. A., Morii, K., Hu, X. L., Negorev, D. G., Spais, C., Ruthig, L. A., and Riethman, H. C. (1995). Molecular cloning and RARE cleavage mapping of human $2 p, 6 q, 8 q, 12 q$, and $18 q$ telomeres. Genome Res. 5: 225-232.

Nicholls, R. D., Fischel-Ghodsian, N., and Higgs, D. R. (1987). Recombination at the human $\alpha$-globin gene cluster: Sequence features and topological constraints. Cell 49: 369-378.

$\mathrm{NIH}$ and Institute of Molecular Medicine Collaboration (1996). A complete set of human telomeric probes and their clinical application. Nat. Genet. 14: 86-89.
Royle, N. J ., Baird, D. M., and J effreys, A. J . (1994). A subterminal satellite located adjacent to telomeres in chimpanzees is absent from the human genome. Nat. Genet. 6: 52-56.

Trask, B. J ., Friedman, C., Martin-Gallardo, A., Rowen, L., Akinbami, C., Blankenship, J ., Collins, C., Giorgi, D., Iadonato, S., J ohnson, F., Kuo, W. L., Massa, H., Morrish, T., Naylor, S., Nguyen, O. T., Rouquier, S., Smith, T., Wong, D. J ., Youngblom, J ., and van den Engh, G. (1998). Members of the olfactory receptor gene family are contained in large blocks of DNA duplicated polymorphically near the ends of human chromosomes. Hum. Mol. Genet. 7: 13-26.

van Deutekom, J . C. T., Lemmers, R. J ., Grewal, P. K., van Geel, M., Romberg, S., Dauwerse, H. G., Wright, T. J., Padberg, G. W., Hofker, M. H., Hewitt, J . E., and Frants, R. R. (1996). Identification of the first gene ( $F R G 1)$ from the FSHD region on human chromosome 4q35. Hum. Mol. Genet. 5: 581-590.

Vergnaud, G. (1989). Polymers of random short oligonucleotides detect polymorphic loci in the human genome. Nucleic Acids Res. 17: 7623-7630.

Vergnaud, G., Mariat, D., Apiou, F., Aurias, A., Lathrop, M., and Lauthier, V. (1991). The use of synthetic tandem repeats to isolate new VNTR loci: Cloning of a human hypermutable sequence. Genomics 11: 135-144.

Wilkie, A. O. M., Higgs, D. R., Rack, K. A., Buckle, V. J ., Spurr, N. K., Fischel-Ghodsian, N., Ceccherini, I., Brown, W. R. A., and Harris, P. C. (1991). Stable length polymorphism of up to $260 \mathrm{~kb}$ at the tip of the short arm of human chromosome 16. Cell 64: 595- 606.

Wong, A. C. C., Ning, Y., Flint, J., Clark, K., Dumanski, J. P., Ledbetter, D. H., and McDermid, H. E. (1997). Molecular characterization of a 130-kb terminal microdel etion at 22q in a child with mild mental retardation. Am. J . Hum. Genet. 60: 113-120. 